Archived version from NCDOCKS Institutional Repository http://libres.uncg.edu/ir/asu/

\title{
Appalacȟnan
}

B O O NE, NORTH CAROLINA

\section{Short-Duration Mechanical Ventilation Enhances Diaphragmatic Fatigue Resistance but Impairs Force Production}

\author{
Authors \\ R. Andrew Shanely, Jeff S. Coombes, A. Murat Zergeroglu, Alistair I. Webb, and Scott K. Powers
}

\begin{abstract}
Study objectives: Mechanical ventilation (MV) is a life-support measure for patients who cannot maintain adequate alveolar ventilation. Following prolonged MV, difficulty in weaning patients from the ventilator can occur, and it has been postulated that difficult weaning is linked to respiratory muscle dysfunction. We tested the hypothesis that $18 \mathrm{~h}$ of controlled MV will diminish diaphragmatic maximal tetanic specific tension (force per cross-sectional area of muscle) without impairing diaphragmatic fatigue resistance.

Design: To test this postulate, adult Sprague-Dawley rats were randomly classified into one of two experimental groups: (1) control group $(n=8)$, and (2) 18-h MV group $(n=6)$. MV-treated animals were anesthetized, tracheostomized, and received room air ventilation. Animals in the control group were acutely anesthetized but did not receive MV. Muscle strips from the mid-costal diaphragm were removed from both experimental groups, and contractile properties were studied in vitro to determine the effects of MV on diaphragmatic endurance and maximal force production. Diaphragmatic endurance was investigated by measuring tension development during repeated contractions throughout a 30-min fatigue protocol.

Results: MV resulted in a reduction (p 0.05) in diaphragmatic maximal specific tension (control group, $26.8 \pm 0.2$ Newtons/cm2 vs MV group, $21.3 \pm 0.6 \mathrm{Newtons} / \mathrm{cm} 2)$. Compared to the control group, diaphragms from MVtreated animals maintained higher ( $\mathrm{p}$ 0.05) percentages of the initial force production throughout the fatigue protocol. The observed improvement in fatigue resistance was associated with an increase in diaphragmatic oxidative and antioxidant capacity as evidenced by increases ( $\mathrm{p} 0.05$ ) in both citrate synthase and superoxide dismutase activities. However, by comparison to the control group, diaphragms from MV-treated animals generated less ( $\mathrm{p}$ 0.05) absolute specific force throughout the fatigue protocol.

Conclusions: These data indicate that $18 \mathrm{~h}$ of MV enhances diaphragmatic fatigue resistance but impairs diaphragmatic specific tension.
\end{abstract}

R. Andrew Shanely, Jeff S. Coombes, A. Murat Zergeroglu, Alistair I. Webb, and Scott K. Powers (2003) "Short-Duration Mechanical Ventilation Enhances Diaphragmatic Fatigue Resistance but Impairs Force Production" Chest 123:195-201 Version of Record available @ (doi:10.1378/chest.123.1.195) 


\title{
Short-Duration Mechanical Ventilation Enhances Diaphragmatic Fatigue Resistance but Impairs Force Production*
}

\author{
R. Andrew Shanely, MA; Jeff S. Coombes, PhD; A. Murat Zergeroglu, MD, PhD; \\ Alistair I. Webb, DVM, PhD; and Scott K. Powers, PhD, EdD
}

\begin{abstract}
Study objectives: Mechanical ventilation (MV) is a life-support measure for patients who cannot maintain adequate alveolar ventilation. Following prolonged MV, difficulty in weaning patients from the ventilator can occur, and it has been postulated that difficult weaning is linked to respiratory muscle dysfunction. We tested the hypothesis that $18 \mathrm{~h}$ of controlled MV will diminish diaphragmatic maximal tetanic specific tension (force per cross-sectional area of muscle) without impairing diaphragmatic fatigue resistance.

Design: To test this postulate, adult Sprague-Dawley rats were randomly classified into one of two experimental groups: (1) control group $(n=8)$, and (2) 18-h MV group $(n=6)$. MV-treated animals were anesthetized, tracheostomized, and received room air ventilation. Animals in the control group were acutely anesthetized but did not receive MV. Muscle strips from the midcostal diaphragm were removed from both experimental groups, and contractile properties were studied in vitro to determine the effects of MV on diaphragmatic endurance and maximal force production. Diaphragmatic endurance was investigated by measuring tension development during repeated contractions throughout a 30 -min fatigue protocol.

Results: MV resulted in a reduction $(\mathrm{p}<0.05)$ in diaphragmatic maximal specific tension (control group, $26.8 \pm 0.2$ Newtons $/ \mathrm{cm}^{2}$ vs MV group, $\left.21.3 \pm 0.6 \mathrm{Newtons} / \mathrm{cm}^{2}\right)$. Compared to the control group, diaphragms from MV-treated animals maintained higher $(p<0.05)$ percentages of the initial force production throughout the fatigue protocol. The observed improvement in fatigue resistance was associated with an increase in diaphragmatic oxidative and antioxidant capacity as evidenced by increases $(p<0.05)$ in both citrate synthase and superoxide dismutase activities. However, by comparison to the control group, diaphragms from MV-treated animals generated less $(p<0.05)$ absolute specific force throughout the fatigue protocol.

Conclusions: These data indicate that $18 \mathrm{~h}$ of MV enhances diaphragmatic fatigue resistance but impairs diaphragmatic specific tension.
\end{abstract}

Key words: fiber type; muscular endurance; respiratory muscles

Abbreviations: $\mathrm{CS}=$ citrate synthase $; \mathrm{Cu}-\mathrm{Zn}-\mathrm{SOD}=$ copper zinc-superoxide dismutase; $\mathrm{LDH}=$ lactate dehydrogenase; $\mathrm{Lo}=$ optimal contractile length; $\mathrm{Mn}-\mathrm{SOD}=$ manganese-superoxide dismutase; $\mathrm{MV}=$ mechanical ventilation; specific $\mathrm{Po}=$ maximal force production per cross-sectional area of muscle; $\mathrm{SOD}=$ superoxide dismutase

$\mathbf{M}$ echanical ventilation (MV) is critical for life support in patients who cannot maintain adequate alveolar ventilation. Clinical-applications of MV include respiratory failure, neuromuscular diseases, drug overdoses, and recovery from general

\footnotetext{
${ }^{*}$ From the Departments of Exercise and Sport Sciences, Center for Exercise Science (Mr. Shanely, and Drs. Coombes, Zergeroglu, and Powers), Physiologic Sciences (Dr. Webb), Physiology

(Dr. Powers), University of Florida, Gainesville, FL; and Faculty of Medicine, Department of Sports Medicine (Dr. Zergeroglu), Ankara University, Ankara, Turkey.

This work was supported by grants from the American Lung Association-Florida and the National Institutes of Health (R01 HL62361) awarded to Dr. Powers.
}

anesthetics. ${ }^{1}$ Unfortunately, following prolonged $\mathrm{MV}$, difficulty in weaning patients from the ventilator occurs in many cases. ${ }^{2-4}$ It has been postulated that difficult weaning is often due to respiratory muscle dysfunction due to diaphragmatic force deficits (ie, reduced maximal force production) and/or decreased diaphragmatic fatigue resistance. ${ }^{2-5}$

To date, only three published reports have investi- 
gated the affects of prolonged MV on diaphragm maximal force production in animals. The findings of these studies are consistent and indicate that MV is associated with a significant decrement in diaphragmatic total force generation and maximal tetanic force production per cross-sectional area of muscle (specific Po). For example, recent animal experiments in our laboratory reveal that as little as $12 \mathrm{~h}$ of MV results in a depression in diaphragmatic specific Po, and this force deficit is exacerbated with time on the ventilator. ${ }^{6}$ These findings are corroborated by other studies, ${ }^{7,8}$ that indicate prolonged MV is associated with a diaphragmatic force deficit. Collectively, these results are consistent with the notion that diaphragmatic force deficits may contribute to difficult weaning.

While it is clear that prolonged MV results in a diaphragmatic force deficit, it is unknown if relatively short durations of MV impair diaphragmatic endurance. At present, only one experiment has investigated the effects of prolonged MV on diaphragmatic fatigue resistance. Anzueto et $\mathrm{al}^{7}$ reported that 11 days of controlled MV in baboons resulted in impaired diaphragmatic endurance as indicated by a decline in transdiaphragmatic force production during repeated stimulation of the phrenic nerves. Although this experiment suggests that prolonged MV diminishes diaphragmatic endurance, several unanswered questions remain. For example, in the experiments of Anzueto et al, ${ }^{7}$ a reduction in transdiaphragmatic pressure during phrenic stimulation could occur due to failure within peripheral nerves and/or the diaphragm. It remains unknown if MV promotes alterations in the diaphragm that result in diminished endurance. Furthermore, while long-term (eg, 11 days) MV impairs diaphragmatic endurance, it is unknown whether relatively short-duration $\mathrm{MV}(e g,<24 \mathrm{~h})$ would also impair diaphragmatic endurance. Hence, these unanswered questions form the rationale for the current experiments. Therefore, we examined the effects of relatively short-duration MV on both diaphragmatic fatigue and specific Po. To separate central fatigue from peripheral fatigue, we investigated diaphragm contractile function in vitro using direct (electrical) stimulation of the muscle. Based on preliminary experiments in our laboratory, we hypothesized that $18 \mathrm{~h}$ of MV impairs diaphragmatic specific Po but does not accelerate the rate of diaphragmatic fatigue development.

\section{Materials and Methods}

\section{Experimental Animals and Research Design}

These experiments were approved by the University of Florida animal use committee, and followed the guidelines for animal experiments established by the National Institutes of Health. Healthy, female, young adult (4-month-old) Sprague-Dawley rats were individually housed, fed rat chow and water ad libitum, and were maintained on a 12-h light/dark cycle for 3 weeks prior to initiation of these experiments. Animals were randomly assigned to one of two experimental groups: control animals $(n=8)$ or MV-treated animals $(n=6)$.

Protocol for Control Animals: The animals in the control group were free of intervention prior to measurement of diaphragmatic contractile properties. That is, these animals did not receive mechanical ventilation or long-term anesthesia prior to study. At the specified time, animals in the control group received an intraperitoneal injection of sodium pentobarbital $(50 \mathrm{mg} / \mathrm{kg}$ body weight). After a surgical plane of anesthesia was achieved, the diaphragm was rapidly removed for measurement of in vitro contractile properties.

Protocol for MV-Treated Animals: All surgical procedures were performed using aseptic technique. Animals randomly selected for MV were anesthetized with an intraperitoneal injection of sodium pentobarbital (50 mg/ $\mathrm{kg}$ body weight). After reaching a surgical plane of anesthesia, the animals were tracheostomized and placed on MV using a volume-driven, small-animal ventilator (model CIV-101; Columbus Instruments; Columbus, OH). All breaths were delivered by the ventilator; hence, this mode of ventilation (ie, controlled MV) results in complete diaphragmatic inactivity. The tidal volume was established at approximately 1 $\mathrm{mL} / 100 \mathrm{~g}$ of body weight with a respiratory rate of 80 breaths / min. Additionally, positive end-expiratory pressure of $1 \mathrm{~cm} \mathrm{H}_{2} \mathrm{O}$ was used throughout the protocol. Previous experiments in our laboratory indicate that this MV protocol results in arterial blood gas and $\mathrm{pH}$ homeostasis during a $24-\mathrm{h}$ period. ${ }^{6}$

A venous catheter was placed in the jugular vein to permit the infusion of isotonic saline solution and sodium pentobarbital when necessary. Anesthesia was maintained over the entire period of MV by infusing sodium pentobarbital $(10 \mathrm{mg} / \mathrm{kg}$ body weight/h). Body temperature was maintained at approximately $37^{\circ} \mathrm{C}$ by use of a recirculating heating blanket. Additionally, heart rate and electrical activity of the heart were monitored via a lead II ECG using needle electrodes placed subcutaneously.

Throughout MV, animals received enteral nutrition using the American Institute of Nutrition-76 rodent diet (Research Diets; New Brunswick, NJ). Furthermore, body fluid homeostasis was maintained via the administration of $2.0 \mathrm{~mL} / \mathrm{kg} / \mathrm{h}$ IV electrolyte solution.

Continuing care during MV included expressing the bladder, removing airway mucus, lubricating the eyes, rotating the animal, and passive movement of the limbs. This care was maintained throughout the experimental period at 2-h intervals. On completion of $\mathrm{MV}$, one segment of the costal diaphragm was removed for measurement of in vitro contractile properties and another segment was dissected, frozen in liquid nitrogen, and stored at $-80^{\circ} \mathrm{C}$ until subsequent assay.

\section{Measurement of In Vitro Diaphragmatic Contractile Properties}

Description of the Muscle Organ Bath: Details of our in vitro protocol have been described in detail elsewhere, ${ }^{9-11}$ and will be briefly described here. After reaching a surgical plane of anesthesia, the entire diaphragm was removed and placed in a dissecting chamber containing a Krebs-Hensleit solution equilibrated with a $95 \% \mathrm{O}_{2} / 5 \% \mathrm{CO}_{2}$ gas. A muscle strip, including the tendenous attachments at the central tendon and rib cage (dimensions, $20 \pm 3 \mathrm{~mm}$ ), was dissected from the midcostal region. The strip was suspended vertically between two lightweight Plexiglas clamps with one end connected to an isometric force transducer (model FT-03; Grass Instruments; Quincy, MA) within a jacketed tissue bath. The force output was recorded via 
a computerized data-acquisition system (Super Scope II; GW Instruments; Somerville, MA; Apple Computer; Cupertino, CA). The tissue bath was filled with Krebs-Hensleit saline solution and $12 \mu, \mathrm{M}$ d-tubocurarine to produce complete neuromuscular blockade. The saline solution within the bath was aerated with gas $\left(95 \% \mathrm{O}_{2} / 5 \% \mathrm{CO}_{2}\right)$, pH was maintained at 7.4 , and the osmolality of the bath was approximately $290 \mathrm{mOsmol}$.

Determination of the Optimal Length/Tension Relationship: Following a 15 -min equilibration period $\left(25^{\circ} \mathrm{C}\right)$, in vitro diaphragmatic contractile measurements were made. The muscle strip was stimulated along its entire length with platinum wire electrodes (modified S48 stimulator; Grass Instruments) using supramaximal (approximately 150\%) stimulation voltage. Opti- mum contractile length (Lo) was determined by systematically adjusting the length of the muscle using a micrometer while evoking single twitches. Thereafter, all contractile properties were measured isometrically at Lo.

Peak Twitch and Tetanic Tension: After finding Lo, the bath temperature was increased to $37^{\circ} \mathrm{C}$; following a 30 -min equilibration period, peak twitch tension was determined using supra- maximal monophasic pulses of $2 \mathrm{~ms}$. Peak isometric twitch tension was determined from a series of three single pulses. Maximal isometric tetanic contractions were then produced using a supramaximal stimulus train of 500-ms duration $(160 \mathrm{~Hz})$. Force was monitored by the computerized system described above. Peak isometric tetanic tension was measured from a series of three contractions with a 3-min recovery between measurements.

Fatigue Tolerance: Five minutes after completion of the last peak tetanic tension measurement, the diaphragm strip was subjected to a 30-min fatigue protocol. To test fatigue resistance, the strip was stimulated submaximally at 2 -s intervals $(30 \mathrm{~Hz}$; 250 -ms train duration) for $30 \mathrm{~min}$. Tolerance to fatigue (fatigue index) was assessed by the percentage of initial force generated by the strip compared to the tension developed at various time intervals during the protocol.

After completion of the contractile measurements, Lo was measured and the diaphragm muscle strip was weighed. Muscle cross-sectional area was determined using the method described by Close, ${ }^{12}$ and this calculation was used to normalize muscle force production to the cross-sectional area of the muscle (ie, specific Po).

\section{Biochemical Measurements}

To determine the effects of MV on both bioenergetic and antioxidant enzymes, we measured the activities of citrate synthase (CS), lactate dehydrogenase (LDH), and superoxide dismutase (SOD). CS is a Krebs cycle enzyme and is considered to be a standard marker of the oxidative capacity of a tissue since it is highly correlated with mitochondrial respiratory capacity. ${ }^{13}$ $\mathrm{LDH}$ is a glycolytic enzyme and is highly correlated with maximal glycolytic flux. ${ }^{13}$ SOD scavenges superoxide radicals and is considered to be one of the most important enzymes in the antioxidant defense system. ${ }^{14} \mathrm{SOD}$ exists in two isoforms in muscle fibers: copper zinc-SOD [CU-Zn-SOD], and manganeseSOD [Mn-SOD]. Cu-Zn-SOD is located in the cytosol, whereas Mn-SOD is found primarily in mitochondria. To determine muscle enzyme activities, muscle samples were homogenized in cold $100-\mathrm{mM}$ phosphate buffer with $0.05 \%$ bovine serum albumin (1:20 weight/volume; $\mathrm{pH} 7.4)$. Homogenates were then centrifuged at $4^{\circ} \mathrm{C}$ for $10 \mathrm{~min}$ at $400 \mathrm{~g}$. The resulting supernatant was decanted and assayed for enzyme activity and protein content. CS, LDH, and SOD activities were measured using techniques described by Srere, ${ }^{15}$ Passonneau and Lowry, ${ }^{16}$ and Quick et al, ${ }^{17}$ respectively. Protein concentration was determined using techniques described by Bradford..$^{18}$

\section{Postmortem Examination}

To determine if our animals acquired an infection during our experiments, three separate blood samples were removed, smeared on microscope slides, and stained using a bacteriologic stain (Gram stain; Sigma Chemical Company; St. Louis, MO); each sample was then microscopically examined for the presence of bacteria. Furthermore, lungs were visually examined for abnormalities. If macroscopic abnormalities of the lung were observed during necropsy, these animals were eliminated from the study.

\section{Statistical Analysis}

Comparisons between groups for each dependent variable measured were made. Isometric contractile parameters and biochemical measurements were analyzed with an unpaired $t$ test, and where significant differences existed a Scheffe test was used post hoc. The fatigue data were analyzed by a two-way analysis of variance with repeated measures across time and analyzed post hoc with a Bonferroni-corrected $t$ test. Significance was established at $\mathrm{p}<0.05$.

\section{Results}

\section{Systemic and Biological Response to MV}

No animals were eliminated due to infection. However, one animal from the MV group was eliminated from our analysis because the postmortem examination revealed evidence of lung barotrauma. Of the animals included in our analysis, initial and final body weights did not differ $(p>0.05)$ between control animals and MV-treated animals. Importantly, $18 \mathrm{~h}$ of MV did not result in a significant change $(p>0.05)$ in animal body weight (data not shown). These results indicate that our schedule of nutrition and rehydration was adequate. Also, animals in the MVtreated group urinated and experienced intestinal transit throughout the experimental period.

Since sepsis promotes diaphragmatic contractile dysfunction, strict aseptic techniques were followed throughout the experiments. Importantly, our results indicate that the MV-treated animals did not acquire infections during MV. For example, microscopic examination of blood revealed no detectable bacteria, and postmortem examination of the lungs (visual) and peritoneal cavity (visual) displayed no detectable abnormalities. Furthermore, our MVtreated animals were afebrile during the investigation, with body temperatures ranging from 36.5 to $37.6^{\circ} \mathrm{C}$; and, as noted above, no significant $(p>0.05)$ changes occurred in the body weights of our MV-treated animals. Collectively, these results indicate that the MVtreated animals used in our analysis were free of significant infection.

\section{MV and Diaphragmatic Contractile Properties}

Loranged from 1.9 to $2.1 \mathrm{~cm}$ across all diaphragm strips investigated, and no differences existed 
Table 1-Maximal Isometric Twitch and Tetanic Force Production in Diaphragm Strips Obtained From Control Animals or MV-Treated Animals*

\begin{tabular}{lccc}
\hline \hline Experimental Groups & $\begin{array}{c}\text { Maximal Isometric Twitch } \\
\text { Force, Newtons } / \mathrm{cm}^{2}\end{array}$ & $\begin{array}{c}\text { Maximal Isometric Tetanic } \\
\text { Force, Newtons/cm }{ }^{2}\end{array}$ & $\begin{array}{c}\text { Twitch/Tetanic } \\
\text { Force Ratio }\end{array}$ \\
\hline Control animals & $5.3 \pm 0.2$ & $26.8 \pm 0.2$ & $0.20 \pm 0.01$ \\
MV-treated animals & $3.7 \pm 0.2 \dagger$ & $21.3 \pm 0.6 \dagger$ & $0.18 \pm 0.01$ \\
\hline
\end{tabular}

*Data are presented as mean \pm SEM.

$\uparrow$ Different from control at $\mathrm{p}<0.05$.

$(p>0.05)$ in Lo between the control group and the MV group. MV resulted in a significant $(p<0.05)$ reduction in both diaphragmatic maximal twitch force production and specific Po (Table 1). In contrast, no group differences ( $p>0.05$ ) existed in the twitch/tetanic force production ratio (Table 1).

Figure 1 illustrates the change in the percentage of initial force production during the 30-min fatigue protocol. Compared to control animals, diaphragms from MV-treated animals maintained a significantly greater $(\mathrm{p}<0.05)$ fatigue index (ie, higher relative forces) throughout the fatigue test. In contrast, when diaphragmatic force production during the fatigue test is compared by the absolute specific force produced, diaphragms from control animals produced higher $(\mathrm{p}<0.05)$ specific forces than diaphragms from MV-treated animals throughout the 30-min fatigue protocol (Fig 2).

\section{Biochemical Measurements}

Activities for CS, LDH, and SOD are contained in Table 2. Compared to MV-treated animals, LDH activities tended to be higher in diaphragms from control animals but these differences were not sig-

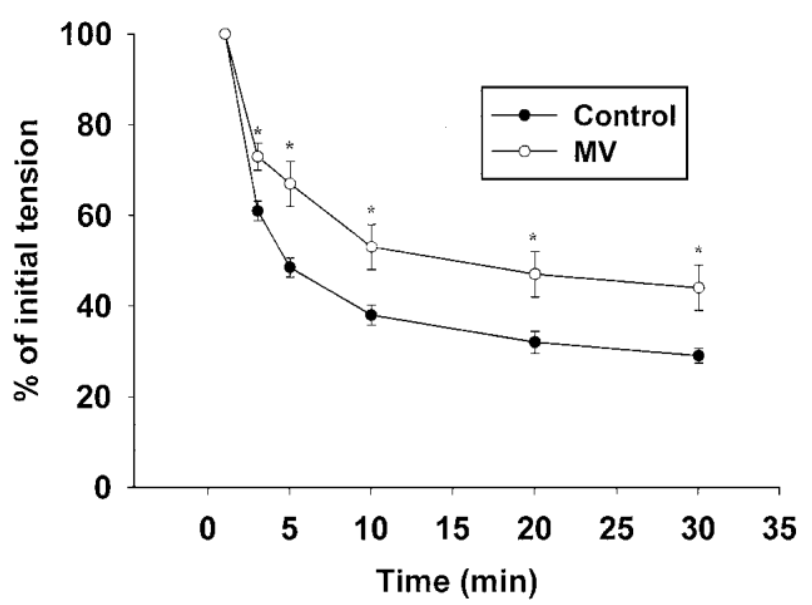

Figure 1. Comparison of the loss of relative force production of the diaphragm during the 30-min fatigue test in both control and MV-treated animals. Values are means \pm SEM. *Group differences at $\mathrm{p}<0.05$. nificant. In contrast, costal diaphragm CS, total SOD, Cu-Zn-SOD, and Mn-SOD activities were significantly higher in MV-treated animals compared to control animals. Our finding that MV elevates diaphragmatic CS differs from a previous report indicating that $48 \mathrm{~h}$ of MV does not alter diaphragmatic oxidative capacity. ${ }^{8}$ The explanation for these divergent findings is not clear.

\section{Discussion}

Overview of Principle Findings

Although previous experiments have reported that relatively short-duration MV results in diaphragmatic force deficits, the present experiments provide important new information regarding the influence of controlled MV on diaphragmatic endurance. Our results support the hypothesis that $18 \mathrm{~h}$ of MV results in a significant reduction (approximately 20\%) in diaphragmatic specific Po. Furthermore, these data support the notion that $18 \mathrm{~h}$ of MV does not accelerate the rate of diaphragmatic fatigue development. In fact, our findings indicate that $18 \mathrm{~h}$

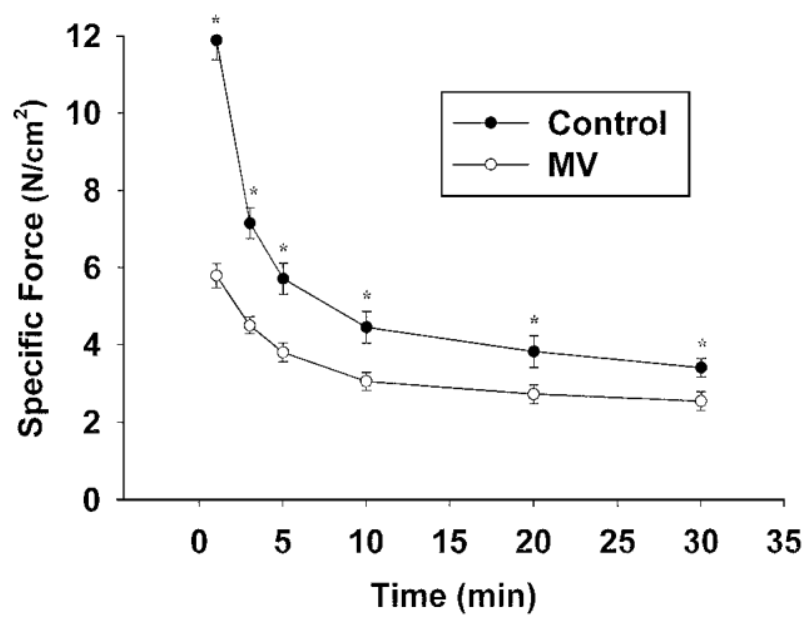

Figure 2. Comparison of diaphragmatic specific force production between control animals and the MV-treated animals. Values are means \pm SEM *Group differences at $\mathrm{p}<0.05 . \mathrm{N}=$ Newton. 
Table 2-CS, LDH, and SOD Activities in the Costal Diaphragm of Control Animals and MV-Treated Animals*

\begin{tabular}{llcccc}
\hline \hline \multicolumn{1}{c}{ Groups } & CS Activity $\dagger$ & LDH Activity $\dagger$ & Total SOD $\ddagger$ & Cu-Zn-SOD $\$$ & Mn-SOD $\$$ \\
\hline Control animals & $0.21 \pm 0.01$ & $2.5 \pm 0.1$ & $29.5 \pm 0.2$ & $11.2 \pm 0.2$ & $18.3 \pm 0.2$ \\
MV-treated animals & $0.33 \pm 0.02 \S$ & $2.2 \pm 0.3$ & $37.2 \pm 0.3 \S$ & $13.4 \pm 0.2 \S$ & $23.4 \pm 0.2 \S$ \\
\hline
\end{tabular}

*Data are presented as mean \pm SEM.

$\dagger$ Expressed as micromoles of substrate converted per minute per milligram of protein.

$\$$ Expressed as units per milligram of protein.

$\S$ Different from control at $\mathrm{p}<0.05$.

of MV improves diaphragmatic fatigue resistance as evidenced by the higher fatigue index in diaphragms of MV-treated animals compared to control animals (Fig 1). A detailed analysis of our experimental model and these findings follows.

\section{Analysis of Experimental Model}

We choose an animal model to investigate the effects of MV on the diaphragm due to the invasive nature of obtaining a diaphragmatic biopsy from human subjects. Our considerations in the choice of an animal model included both pragmatic issues (ie, animal size and cost) and the applicability of the animal model to humans. In regard to pragmatic issues, the rat is a relatively inexpensive model to study the effects of MV, and does not pose technical difficulties during tracheostomy and enteral feeding. In reference to applicability to humans, the rat is an excellent model to investigate diaphragmatic issues since human and rat diaphragms are similar in muscle fiber type, gross anatomic features, and function. . $^{13,19}$

To investigate the effects of prolonged MV on diaphragmatic contractile function, we chose to use controlled MV for two principal reasons. Firstly, compared to assist modes of MV, an advantage of controlled MV is that this mode results in rapid diaphragmatic atrophy. ${ }^{20}$ This is experimentally advantageous because it permits the study of the adverse effects of MV on the diaphragm following relatively short periods of MV. Secondly, although many patients receiving MV have some form of pressure-assist ventilation, controlled MV has clinical relevance because this mode of ventilation is used in adult patients in several circumstances (eg, drug overdose, spinal cord injury, surgery, etc). ${ }^{1}$ Further- more, controlled MV is also used widely in pediatric situations. ${ }^{1}$

We selected sodium pentobarbital as the general anesthetic in these experiments because this anesthetic, when used in low doses, does not negatively impact locomotor and diaphragm contractile function. For example, work by Le Bourdelles et $\mathrm{al}^{8}$ reveals that the level of sodium pentobarbital required to maintain a surgical plane of anesthesia in rats over a 48-h period does not promote locomotor muscle atrophy nor does it impair locomotor muscle maximal tetanic force production. Furthermore, recent experiments in our laboratory with anesthetized, spontaneously breathing animals clearly reveals that the level of sodium pentobarbital required to maintain a surgical plane of anesthesia does not impair diaphragm contractilefunction following $24 \mathrm{~h}$ of anesthesia. ${ }^{6}$

\section{Prolonged MV and Diaphragmatic Force Deficits}

The current experiments support previous reports $^{6-8}$ that prolonged MV results in a significant reduction in both twitch and maximal diaphragmatic specific Po. There are at least four possible explanations for the diaphragmatic force deficit associated with prolonged MV. These include the negative impact of anesthesia on muscle function, infection, arterial $\mathrm{pH}$ imbalance, and/or intrinsic alterations in diaphragm muscle fibers. ${ }^{21-24}$ Previous experiments in our laboratory indicate that the MV-induced diaphragmatic contractile dysfunction is not due to anesthesia, infection, or a $\mathrm{pH} /$ blood gas disturbance. ${ }^{6}$ Therefore, we have concluded that the MV-induced contractile dysfunction is due to intrinsic changes within diaphragmatic muscle fibers $^{6}$ that could theoretically stem from one or several different mechanisms. Specifically, a reduction in myofibrillar protein concentration, abnormalities of contractile/ cytoskeletal proteins, and/or impaired excitation contraction-coupling could individually or collectively contribute to the observed force deficit. $9,10,24,25$ Which of these factors is responsible for the observed force deficit is unknown and warrants further study.

\section{Moderate-Duration MV Improves Diaphragmatic Fatigue Resistance}

Our results indicate that $18 \mathrm{~h}$ of MV improves diaphragmatic resistance to fatigue (Fig 1). At least four different physiologic mechanisms could explain this observation. First, MV is associated with an increase in diaphragmatic oxidative capacity. This prospect was supported by our experimental findings 
that CS activity (Krebs cycle enzyme and a marker of oxidative capacity) in the diaphragm was significantly higher in MV-treated animals compared to control animals (Table 2). This group difference in diaphragmatic CS activity is likely related to the pattern of muscle fiber atrophy during MV. A previous study in our laboratory indicates that while MV results in atrophy of all fiber types within the diaphragm, compared to type I fibers, the magnitude of MV- induced atrophy was greatest in type IIx and type IIb fibers. ${ }^{20}$ In our previous study, the relative contribution of these type II fibers to the total cross-sectional area of the diaphragm was approximately $53 \%$ in the control animals and was reduced to approximately $46 \%$ in the MV-treated animals. This is significant because type IIx and type IIb fibers possess the lowest oxidative capacity and are therefore the most fatigable motor units in the diaphragm. Hence, a selective atrophy of type IIx and type IIb fibers would reduce the relative rate of fatigue in diaphragms from MV-treated animals. ${ }^{26}$ We postulate that this explanation accounts for a significant portion of the MV-induced improvement in diaphragmatic fatigue resistance. Similar conclusions have been reached by others investigating the effects of malnutrition on diaphragmatic fatigue. ${ }^{26}$

A second explanation for the improved diaphragmatic fatigue resistance associated with MV is the finding that diaphragms from MV-treated animals possessed elevated SOD activities. This is significant because SOD scavenges superoxide radicals and is considered to be one of the most important enzymes in the antioxidant defense system. ${ }^{14}$ Furthermore, it is noteworthy that the activities of both intracellular isoforms of SOD (ie, Cu-Zn-SOD and Mn-SOD) were elevated in diaphragms from MV-treated animals. This observation indicates that MV is associated with improved superoxide scavenging capacity in both the cytosol and mitochondria. The biological importance of an elevated antioxidant capacity is that contraction-induced radical production and oxidative stress in the diaphragm is a contributory factor to diaphragmatic fatigue. ${ }^{27-}$ ${ }^{29}$ Hence, improved antioxidant capacity in the diaphragms of MV-treated animals could counteract contraction-induced oxidant stress and improve diaphragmatic fatigue resistance.

A third potential explanation for the enhanced fatigue resistance of diaphragms from MV-treated animals relates to the fact that MV-induced atrophy is associated with a reduction in muscle fiber crosssectional area. ${ }^{20}$ This reduction in fiber crosssectional area reduces the distances for substrate diffusion within muscle fibers. Hence, this atrophyrelated decrease in diffusion distances for energy substrates could facilitate substrate utilization and energy production. ${ }^{26}$ Nonetheless, at present, it is unknown if diaphragmatic atrophy alone will improve fatigue resistance.

A final possible explanation for the enhanced fatigue resistance of diaphragms from MV-treated animals relates to the observation that $\mathrm{MV}$ is associated with a reduction in diaphragmatic force production per cross-sectional area of the fiber (ie, reduced specific force production). This observation suggests that adenosine triphosphate utilization would be reduced in diaphragms from MV animals. Hence, a decrease in the rate of adenosine triphosphate utilization in muscle fibers could lead to a slower rate of fatigue.

While the current experiments demonstrate that short-duration MV improves diaphragmatic fatigue resistance, it seems likely that long duration MV (ie, weeks to months) will result in a significant decline in both diaphragmatic oxidative capacity and fatigue resistance. Indeed, experiments investigating the physiologic effects of unloading locomotor muscles indicate that 15 days of hind-limb suspension results in increased fatigability in the soleus muscle. ${ }^{30}$ Further, work by Anzueto et $\mathrm{al}^{7}$ demonstrated that 11 days of MV resulted in a decrease in diaphragmatic fatigue resistance in baboons. Therefore, it seems likely that while relatively short-duration MV does not impair diaphragmatic fatigue resistance, long duration MV (weeks-to-months) would impair diaphragmatic endurance.

\section{Functional Significance of MV-Induced Changes in Diaphragmatic Contractile Function}

While the improved fatigue resistance observed in diaphragms from MV-treated animals would appear to be beneficial against fatigue, it is important to consider this in the context of the decrement in total specific tension development that occurs following $18 \mathrm{~h}$ of MV (Fig 2). This reduction in total diaphragmatic force production in diaphragms from MV animals would likely place constraints on the diaphragmatic force reserve during periods of increased loads. ${ }^{26}$ Therefore, in clinical situations, diaphragms from MV-treated patients may not be capable of maintaining ventilatory requirements during loading conditions that would occur in patients with obstructive airway diseases. In these circumstances, diaphragmatic fatigue and respiratory failure could occur despite the apparent improvement in the rate of diaphragmatic fatigue development following MV.

\section{References}

1 Hess D, Kacmarek RM. Essentials of mechanical ventilation. New York, NY: McGraw-Hill, Health Professions Division, 1996 
2 Chevrolet JC. Difficult weaning from mechanical ventilation. Lung 1990;168(Suppl):829-832

3 Kelly SM, Rosa A, FieldS, et al. Inspiratory muscle strength and body composition in patients receiving total parenteral nutrition therapy. Am Rev Respir Dis 1984; 130:33-37

4 Sporn PH, Morganroth ML. Discontinuation of mechanical ventilation. Clin Chest Med 1988; 9:113-126

5 Tobin MJ, Laghi F, Jubran A. Respiratory muscle dysfunction in mechanically-ventilated patients. Mol Cell Biochem 1998; 179:87-98

6 Powers SK, Shanely RA, Coombes JS, et al. Mechanical ventilation results in progressive contractile dysfunction in the diaphragm. J Appl Physiol 2002;92:1851-1858

7 Anzueto A, Peters JI, Tobin MJ, et al. Effects of prolonged controlled mechanical ventilation on diaphragmatic function in healthy adult baboons. Crit Care Med 1997; 25:1187-1190

8 Le Bourdelles G, Viires N, Boczkowski J, et al. Effects of mechanical ventilation on diaphragmatic contractile properties in rats. Am J Respir Crit Care Med 1994; 149:1539-1544

9 Criswell DS, Powers SK, Herb RA, et al. Mechanism of specific force deficit in the senescent rat diaphragm. Respir Physiol 1997; 107:149-155

10 Herb RA, Powers SK, Criswell DS, et al. Alterations in phenotypic and contractile properties of the rat diaphragm: influence of hypothyroidism. J Appl Physiol 1996; 80:21632170

11 Powers SK, Criswell D, Herb RA, et al. Age-related. increases in diaphragmatic maximal shortening velocity. J Appl Physiol 1996; 80:445-451

12 Close RI. Dynamic properties of mammalian skeletal muscles. Physiol Rev 1972; 52:129-197

13 Powers SK, Demirel HA, Coombes JS, et al. Myosin phenotype and bioenergetic characteristics of rat respiratory muscles. Med Sci Sports Exerc 1997; 29:1573-1579

14 Powers SK, Ji LL, Leeuwenburgh C. Exercise traininginduced alterations in skeletal muscle antioxidant capacity: a brief review. Med Sci Sports Exerc 1999; 31:987-997

15 Srere P. Citrate synthase. Methods Enzymol 1969; 13:3-5

16 Passonneau JV, Lowry OH. Enzymatic analysis: a practical guide. Totowa, NJ:Humana Press, 1993

17 Quick KL, Hardt JI, Dugan LL. Rapid microplate assay for superoxide scavenging efficiency. J Neurosci Methods 2000; 97:139-144

18 Bradford MM. A rapid and sensitive method for the quantitation of microgram quantities of protein utilizing the principle of protein-dye binding. Anal Biochem 1976; 72:248-254

19 Mizuno M. Human respiratory muscles: fibre morphology and capillary supply. Eur Respir J 1991; 4:587-601

20 Shanely RA, Zergeroglu AM, Lennon SL, et al. Mechanical ventilation-induced diaphragmatic atrophy is associated with oxidative injury and increased proteolytic activity. Am J Respir Crit Care Med 2002; 166:1369-1374

21 BoczkowskiJ, Dureuil B, Branger C, etal. Effects of sepsis on diaphragmatic function in rats. Am Rev Respir Dis 1988; 138:260-265

22 Coast JR, Shanely RA, Lawler JM, et al. Lactic acidosis and diaphragmatic function in vitro. Am J Respir Crit Care Med 1995; 152 (5 pt 1):1648-1652

23 Taylor RG, Abresch RT, Lieberman JS, et al. Effect of pentobarbital on contractility of mouse skeletal muscle. Exp Neurol 1984; 83:254-263

24 Reid MB. Handbook of oxidants and antioxidants in exercise. Amsterdam, The Netherlands: Elsevier, 2000;599-630

25 Horowits R, Kempner ES, Bisher ME, et al. A physiological role for titin and nebulin in skeletal muscle. Nature 1986; 323:160-164

26 SieckGC, Lewis MI, Blanco CE. Effects of undernutrition on diaphragm fiber size, SDH activity, and fatigue resistance. J Appl Physiol 1989; 66:2196-2205

27 Reid MB, Haack KE, Franchek KM, et al. Reactive oxygen in skeletal muscle: I. Intracellular oxidant kinetics and fatigue in vitro. J Appl Physiol 1992; 73:1797-1804

28 Diaz PT, She ZW, Davis WB, et al. Hydroxylation of salicylate by the in vitro diaphragm: evidence for hydroxyl radical production during fatigue. J Appl Physiol 1993; 75:540-545

29 Shindoh C, DiMarco A, Thomas A, et al. Effect of N-acetylcysteine on diaphragm fatigue. J Appl Physiol 1990; 68:21072113

30 McDonald KS, Delp MD, Fitts RH. Fatigability and blood flow in the rat gastrocnemius-plantaris-soleus after hind-limb suspension.J Appl Physiol1992;73:1135-1140 